\title{
ASSESSMENT OF LOW PEAK KILO VOLTAGE, ELECTROCARDIOGRAM GATED MULTI-DETECTOR COMPUTED TOMOGRAPHY IN CONGENITAL HEART DISEASE AT PEDIATRIC AGE GROUP
}

\author{
Dina Hamdy Ahmed*; Abeer Maghwary Abd Elhameed; Mohamed Amin Nassef; \\ Susan Adil Ali Abdul Rahim and Emad Hamid Abdeldayem
}

\footnotetext{
* Radio diagnosis department, Ain Shams University, Cairo, Egypt * Radio diagnosis specialist, Ministry Of Health, Cairo, Egypt $^{2}$.

Corresponding author:

Dina Hamdy Ahmed

Mobile: 01005648788

E mail:

dina1186@windowslive.com

Received: 8/4/2019

Accepted:21/4/2019
}

\begin{abstract}
Back ground: Congenital heart disease (CHDs) is the most common birth anomaly worldwide and it has a significantly increasing prevalence rate. Traditional evaluation for assessment depends on echocardiography (ECHO) and conventional angiography. Recent technological advances in computed tomography $(C T)$, is increasingly used for noninvasive evaluation and proper diagnosis.
\end{abstract}

Aim of work: To examine the validity of administering low exposure dose by MDCT with the aid of ECG gating technique and post processing $3 D$ reconstruction in diagnosis and follow up of congenital heart disease in pediatrics.

Patients \& methods: This study was performed on 40 patients with age ranged from 3 days to 16 year old with suspicious or clinically /echocardiographically known to have congenital heart disease. The study was performed with MDCT machines (64 and 80 slices spiral CT)with ECG gating technique. The radiation dose was kept to minimum by reducing the kilo voltage to $80 \mathrm{kvp}$. All patients underwent Nonionic contrast agent injection.

Results: we found that there is overall no significant difference between the low Kvp ECG gated MDCT and high Kvp MDCT in detecting congenital cardiac anomalies moreover, in our study we detected some anomalies which were not reported by high Kvp protocol in other studies.

Conclusion: low Kvp ECG gated MDCT is superior to conventional method of CT scanning in assessment of congenital heart anomalies as it reaches to the same diagnosis with less radiation dose and hazards

Key words: ECG gated, congenital, low Kvp, MDCT, pediatrics.

\section{INTRODUCTION:}

Congenital heart diseases affect about $1 \%$ of total births per year in United States of America $^{(\mathbf{1})}$, and considered the cause of 30 $50 \%$ of birth defect mortality in infants and children $^{(2)}$.

Primary evaluation of CHDs traditionally depends on echocardiography (ECHO) and conventional angiography, these two techniques have some limitations such as being operator dependant, insufficient evaluation or proper imaging of great vessels, missing extra cardiac assessment, being invasive in case of angiography with a potential risk for vascular injury, bleeding and infection ${ }^{(3)}$. 
The development of recent advances of multidetector CT (MDCT), is increasingly used in addition to ECHO for noninvasive evaluation of the morphological features of vessels and cardiac chambers in patients with CHDs in a short time ${ }^{(4)}$. For infants with congenital heart disease, electro cardiogram (ECG)-gated cardiac CT angiography (CTA) is the modality of choice for imaging the coronary arteries, airways, cardiac anatomy and extra cardiac vascular structures with its fast scanning times and high-quality images, it aids in patient management and treatment planning ${ }^{(5)}$.

MDCT provides high-quality threedimensional (3D) images using methods such as volume rendering and maximum intensity projection the resulting images are valuable for assessing complex cardiovascular birth defects especially for surgeons $^{(6)}$.

The degree of radiation exposure associated with MDCT is of concern, most notably in pediatric patients as they are more sensitive than adults to the effects of ionizing radiation, therefore, it is essential to balance image quality with radiation dose delivered when performing CTA in children. The dose should be kept as low as reasonably achievable (ALARA) ${ }^{(7)}$. The estimated Radiation doses for prospective and retrospective ECG-gated protocols have been reported to be ranging from $1-10 \mathrm{mSv}$ using low peak kilo voltage and low milliamperes per kilogram ${ }^{(8 \& 9)}$.

Three Dimensional CTA is typically performed with low tube voltages in children because the attenuation of contrast media becomes higher and also it can be easily reconstructed. Moreover, the amount of contrast media can be reduced by using a lower tube voltage ${ }^{(4)}$.

\section{AIM OF THE WORK:}

To examine the validity of administering low exposure dose by MDCT with the aid of ECG gating technique and post processing 3D reconstruction in diagnosis and follow up of congenital heart disease in pediatric patients.

\section{PATIENTS \& METHODS:}

This study included 40 patients (19 females and 21 males with age ranged from 3 days to 16 years old) suspected or clinically/echocardiographically known to have congenital heart disease. referred for MDCT examination, the study was performed in Ain Shams university hospitals \& some private centers in Egypt. The study was performed after approval of the Ethical committee of scientific Research, faculty of medicine, Ain Shams University. Patients were examined in the period from 2016 to 2018.

\section{Patient's preparation:}

A written consent was gathered from all the care givers of all the patients included in the study. History taking, checking renal functions, fasting for at least 3 hours was required prior to the examination, calculation of height, weight $\&$ body surface area for calculation of contrast material injected volume as well as radiation dose.

\section{MDCT imaging protocol}

CT study was performed by using 64/80slice MDCT Toshiba scanner.

Data acquisition was performed from the thoracic inlet level to diaphragm.

\section{Retrospective ECG qating}

Utilizing low dose protocol was performed in all cases complying with ALARA (As Low As Reasonably Achievable) principle. We used ALARA principle in form of reduction of Kvp and automatic adjustment of tube current according to body weight.

\section{Contrast media:}

Non-ionic contrast agent, $(1-2 \mathrm{ml} / \mathrm{kg})$ contrast agent followed by saline $15-20 \mathrm{ml}$, 
injection rate $1.5-3 \mathrm{ml} / \mathrm{s}$ injected by dual mechanical power injector.

Examination of the heart was then performed. Sequential series of images in arterial and subsequent phases of enhancement were taken to ensure opacification of both sides of the heart and all extra-cardiac vessels.

\section{Image analysis:}

The acquired axial images were reconstructed in sagittal and coronal planes. Furthermore a variety of high quality 2D reformatted and $3 \mathrm{D}$ reconstructed images were generated that aided in the understanding of complex cardiovascular anatomy.

MDCT data were reviewed to obtain all clinically relevant information using a combination of three dimensional maximum intensity projections (MIP), volume rendering (VR), multi planar (MPR) and curved planer reformations (CPR), also volumetric \& functional analysis were done.

\section{Statistical methods :}

IBM SPSS statistics (V. 25.0, IBM Corp., USA, 2017-2018) was used for data

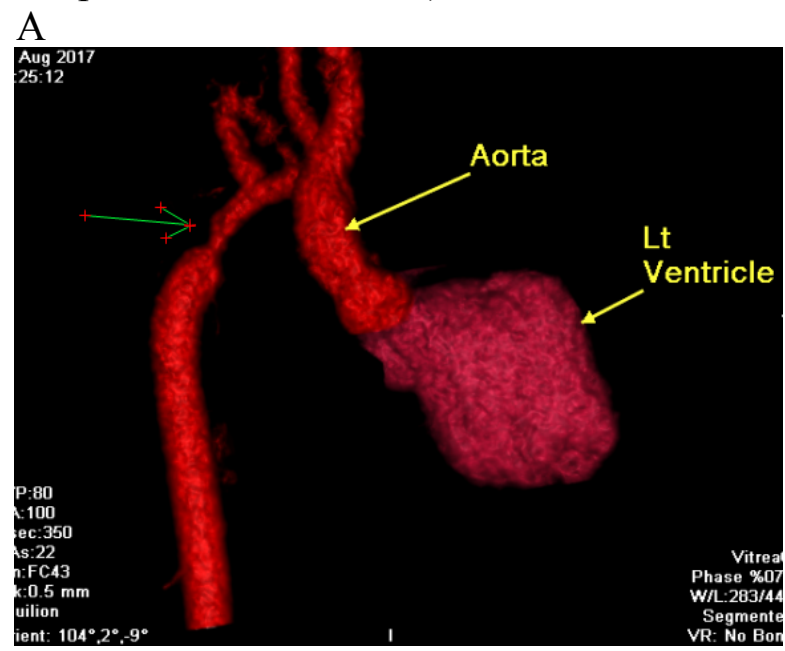

analysis. Data were expressed as both number and percentage for categorized data. Chi-square test was used to study the association between each 2 variables or comparison between 2 independent groups as regards the categorized data. The probability of error at 0.05 was considered significant, while at 0.01 and 0.001 are highly significant and more than 0.05 is non significant.

\section{RESULTS:}

This study was carried out including a total of 40 patients presented with clinically/ echocardiographically known/ suspected to have congenital heart disease. They were 19 $(47.5 \%)$ females and $21(52.5 \%)$ males. Their age ranged from 3 day to 16 years old. As most of patients found to have multiple anomalies, we categorized the results into most common findings and they were 21 findings as shown in (table 1). Illustrated images of MDCT and 3D reconstruction of examples of the findings in the following figure.

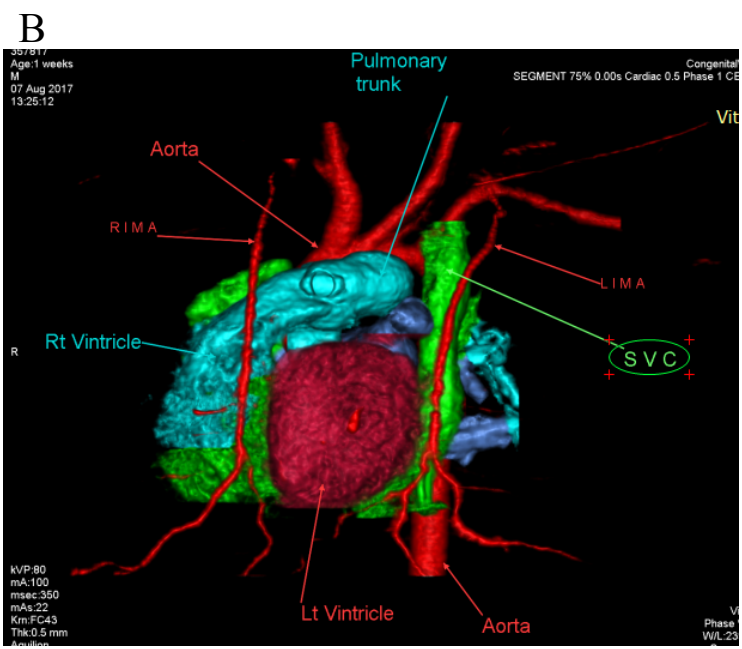



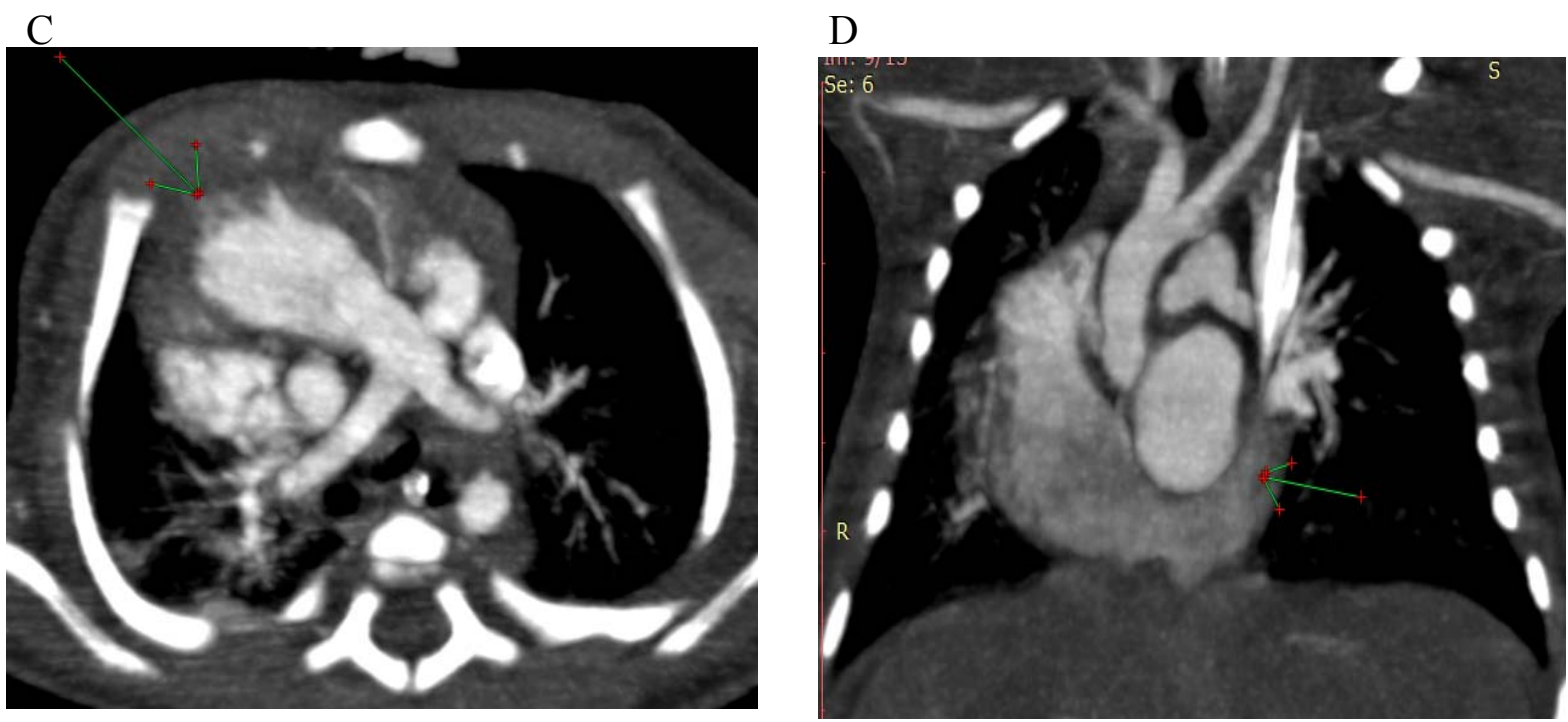

Figure: A case of 13 days old male with multiple congenital heart anomalies (Aortic coarctation, persistent left SVC, dextrocardia and right ventricular hypertrophy):

$\mathrm{A}$ and $\mathrm{B}$ ) 3D reconstruction images ( A: green arrow pointed to aortic coarctaion, B: circled green annotation marked the site of left sided SVC), C) MDCT axial images showing dextrocardia and the green arrow points to the hypertrophied right ventricle, D) MDCT coronal reconstruction : the green arrow points to the left sided SVC

Table 1: the findings detected in our study with its count and incidence percentage in the study sample.

\begin{tabular}{|l|l|c|c|}
\hline Classification & \multicolumn{1}{|c|}{ Finding } & Count & $\%$ \\
\hline \multirow{3}{*}{ Septal anomlaies } & Ventricular Septal Defect (VSD) & 14 & 35 \\
\cline { 2 - 4 } & Atrial Septal Defect (ASD) & 14 & 35 \\
\hline \multirow{5}{*}{ Aortic anomalies } & Aortic Coararctation & 6 & 15 \\
\cline { 2 - 4 } & Right aortic arch & 5 & 12.5 \\
\cline { 2 - 4 } & Over riding of aorta & 5 & 12.5 \\
\cline { 2 - 4 } & Aortic pseudocoarctaion & 2 & 5 \\
\cline { 2 - 4 } & Aortic ectasia & 1 & 2.5 \\
\hline \multirow{3}{*}{$\begin{array}{l}\text { Pulmonary and } \\
\text { great vessels }\end{array}$} & Pulmonary stenosis /atresia & 14 & 35 \\
\cline { 2 - 4 } & Pulmonary dialation & 7 & 17.5 \\
\cline { 2 - 4 } & Pulomanry hypertension & 6 & 15 \\
\cline { 2 - 4 } & Perisitant left svc & 6 & 15 \\
\cline { 2 - 4 } & Anomlaous pulomnary venous return & 3 & 7.5 \\
\cline { 2 - 4 } & Transposition Of Great Vessels (TGA) & 3 & 7.5 \\
\hline \multirow{3}{*}{$\begin{array}{l}\text { Connection } \\
\text { anomalies }\end{array}$} & Patent Ductus Artreiosis (PDA) & 9 & 22.5 \\
\cline { 2 - 4 } & Double Outlet Right Ventricle (DORV) & 5 & 12.5 \\
\hline Valvular Anomales & & 3 & 7.5 \\
\hline \multirow{3}{*}{ Chamber anomalies } & Ventricular dialation & 8 & 20 \\
\cline { 2 - 4 } & Ventricular hypertrophy & 3 & 7.5 \\
\cline { 2 - 4 } & Single ventricle & 3 & 7.5 \\
\cline { 2 - 4 } & Single atrium & 6 & 2.5 \\
\hline Mixed & Fallot tetraology & & 15 \\
\hline
\end{tabular}

We compared the results of our study with other studies using High Kvp MDCT and the outcome of comparison was expressed as (probability of error and difference significance) as illustrated in (table 2)

The blank cells referring to (not compared results) as these findings were not detected at the other comparing studies. 
Table 2: probability of error and difference significance between our study findings and other comparing studies .

\begin{tabular}{|l|l|l|}
\hline \multicolumn{1}{|c|}{ Finding } & P value & Significance \\
\hline VSD & 0.000 & H. S. \\
\hline ASD & 0.000 & H. S. \\
\hline Aortic coararctation & 0.844 & N. S. \\
\hline Right aortic arch & 0.026 & S. \\
\hline Over riding of aorta & 0.685 & N. S. \\
\hline Aortic pseudocoarctaion & 0.325 & N. S. \\
\hline Aortic ectasia & 0.841 & N. S. \\
\hline Pulmonary stenosis /atresia & 0.000 & H. S. \\
\hline Pilmonary dialation & 0.000 & H. S. \\
\hline Pulomanry hypertension & \multicolumn{2}{|l|}{} \\
\hline Perisitant left svc & 0.130 & N. S. \\
\hline Anomlaous pulomnary venous return & 0.304 & N. S. \\
\hline TGA & 0.376 & N. S. \\
\hline DORV & 0.009 & H. S. \\
\hline PDA & 0.028 & S. \\
\hline Valvular anomales & 0.224 & N. S. \\
\hline Ventricular hypertrophy & 0.826 & N. S. \\
\hline Ventricular dialation & \multicolumn{2}{|l}{} \\
\hline Single ventricle & \multicolumn{2}{|l}{} \\
\hline Single atrium & & \multicolumn{1}{|l|}{} \\
\hline Fallot Tetraology & 0.130 & N. S. \\
\hline
\end{tabular}

P value: probability of error, VSD: ventricular septal defect, ASD: atrial septal defect, TGA: transposition of great vessels, SVC: superior vena cava, H.S: highly significant, N.S: non significant, S: significant.

Four findings were detected in our study and not found in other comparing studies: three single ventricle cases with percentage of $7.5 \%$, one single atrium case with percentage of $2.5 \%$, six pulmonary hypertension cases with percentage of $15 \%$ and eight ventricular dilatation cases with percentage of $20 \%$.

\section{DISCUSSION:}

In the last two decades, CT scan and MRI have emerged as worthy non-invasive cardiovascular diagnostic tools efficient in providing accurate anatomic and functional information not obtainable by other imaging modalities currently available ${ }^{(\mathbf{1 0})}$ MDCT has the characteristics of fast scanning speed, high resolution, powerful image postprocessing and clear visualization of the anatomic structures of the cardiac chambers and great vessels ${ }^{(11)}$ The sensitivity and the complications risks associated with radiation exposure of children are greater than that of adults yet decreasing the tube voltage and current can effectively reduce the dose of radiation received by a patient ${ }^{(\mathbf{1 1 )}}$. MDCT is the preferred technique for morphological pre-operative evaluation of the aorta like aortic coarctation and aortic arch anomalies. It accurately identifies the aortic coarctation site, determines the degree of narrowing adding to definition of extent of hypoplasia of the aortic arch aiding in choice of surgical technique performed ${ }^{(12)}$. The introduction of the ECG-synchronized scan with either retrospective gating or prospective triggering is an update for the CT imaging of congenital heart disease. ECG-synchronization enables us to accurately evaluate the coronary arteries, the conotruncal and other intracardiac structures ${ }^{(13)}$.

We compared our results with two similar studies yet using high Kvp dose the first one was by Bayraktutan et al ${ }^{(14)}$ dated 2013 was on 102 paediatric cases suspected 
to have congenital heart disease and the other study was by Al-Mousily et al ${ }^{(\mathbf{9})}$. Dated 2011 was on 22 paediatric cases suspected to have congenital heart disease.

There was high significant difference between our study (low Kvp MDCT) and other studies (high Kvp MDCT) in favor for our study in detecting ventricular septal defect, atrial septal defect, pulmonary stenosis and atresia ,pulmonary dilatation and double outlet right ventricle. That was in agreement with what Shehata et al ${ }^{(15)}$ reported in his study 2016 As regard pulmonary artery anomaly diagnosis by MDCT

There was a significant difference between our study (low Kvp MDCT) and other studies (high Kvp MDCT) in favor for our study in detecting right aortic arch and patent ductus arteriorsis.

That was in agreement with what Seif El Dien et $\mathrm{al}^{\mathbf{( 1 6 )}}$ reported in his study 2014 in depicting cases of PDA by MDCT

There was no significant difference between our study (low Kvp MDCT) and other studies (high Kvp MDCT) in detecting aortic coarctation, aortic pseudo-coarctation, aortic ectasia, over riding of aorta, persistent left superior vena cava, anomalous pulmonary venous return, transposition of great vessels, valvular anomalies, ventricular hypertrophy and fallot tetrology. That was in agreement with what Kamala et al. ${ }^{(17)}$ reported in his study 2011 about diagnosis of the coarctation of the aorta by MDCT . And in agreement with the study of Osama et al (18) done 2013 that MDCT correctly depict the TAPVR (Total anomalous pulmonary venous return) and PAPVR (Partial anomalous pulmonary venous return) types of pulmonary venous anomalies. Regarding great vessels anomalies, we found good agreement with what Shehata et al., ${ }^{(15)}$ reported in his study 2017.

We found an agreement with what $\mathrm{Bu}$ et al. ${ }^{(11)}$ reported in his study done 2016 in assessing of MDCT for the diagnosis of cardiac malformations

Also, In agreement with what Shehata et al. ${ }^{(15)}$ reported in his study done 2017 about The diagnostic function of MDCT in congenital heart defects.

\section{Conclusion}

In our study we used retrospective ECG gating utilizing low dose protocol in all cases complying with ALARA (As Low As Reasonably Achievable) principle. ALARA principle was used in the form of reduction of Kvp to 80 and automatic adjustment of tube current according to body weight. As the majority of paediatric group had high heart rate and difficulties in breathing control so we used retrospective gating to get good quality reconstructed images so as not to miss an abnormality and to avoid repetition of the study and repetitive radiation exposure.

Our aim in this study was to assess the role of ECG gated multidetector Computed Tomography in the detection and characterization of congenital heart diseases.

Conclusion of the study results that low Kvp ECG gated MDCT can detect congenital cardiac anomalies as accurate as high Kvp MDCT, moreover, it can detect more anomalies as discussed, with less radiation hazards .

\section{Limitations:}

There were some limitations in this study; firstly not all patients included in this study were subjected to surgery or catheterization to get a full post- operative data. Second we could not perform both high Kvp and low Kvp MDCT examination to the same patient to compare precisely due to the risk of cumulative high radiation exposure to patients. Third, the sample sizes of the present study as well as the compared studies were relatively small and future studies with greater populations should be considered. 


\section{REFERENCES:}

1. Reller M., Strickland M., Riehle T., Mahle W. and Correa A.: Prevalence of congenital heart defects in Atlanta. J Pediatr, 2008; 153:807-13.

2. Pertini J., Damus K., Russell R., Poschman K., Davidoff M. and Mattison D.: Contribution of birth defects to infant mortality in the United States.Teratology, 2002; 66:S3-S6.

3. Saad M., Rohnean A., Cinqualbre A. , Adler G. and Paul J. : Evaluation of image quality and radiation dose of thoracic and coronary dual-source CT in 110 infants with congenital heart disease. Pediatr Radiol, 2009: 39; 668-676.

4. Nakagawa M., Ozawa Y., Sakurai K., Shimohira M., Ohashi K., Yamaguchi S. and Shibamoto Y.: Image quality at low tube voltage $(70 \mathrm{kV})$ and sinogram-affirmed iterative reconstruction for computed tomography in infants with congenital heart disease. Pediatric Radiology, 2015; 45: 1472-1479.

5. Richardson R. and Chau C.: Scanning Technique for Cardiac CTA in Infants and Small Children in Richardson R. R. (eds): Atlas of Pediatric Cardiac CTA. Springer Science and Business Media New York. 2013: 3-7

6. Itatani R., Oda S. and Utsunomiya D. et al :Reduction in radiation and contrast medium dose via optimization of low-kilo voltage CT protocols using a hybrid iterative reconstruction algorithm at 256slice body CT, phantom study and clinical correlation. Clin Radiol, 2013; 68:e128e135.

7. Paul J., Rohnean A., Elfassy E. and Cinqualbre A.:Radiation dose for thoracic and coronary step-and-shoot CT using a 128-slice dual-source machine in infants and small children with congenital heart disease. Pediatr Radiol , 2011; 41:244-249.

8. Deak P., Smal Y. and Kalender W.: Multisection CT protocols: sex- and agespecific conversion factors used to determine effective dose from dose-length product. Radiology, 2010;257(1):158-66.

9. Al-Mousily F., Shifrin R., Fricker F., Feranec N., Quinn N. and Chandran A.: Use of 320-detector computed tomographic angiography for infants and young children with congenital heart disease. Pediatric Cardiology, 2011; 32(4):426-32.

10. Corno F. and Festa P. :Congenital heart defects decision making for Cardiac surgery, August 2008, volume 3, page 95.

11. Bu G, Miao Y, Bin Y, Deng S, Liu T, Jiang $\mathrm{H}$ and Chen W. : Comparison of 128-Slice Low-Dose Prospective ECG-Gated CT Scanning and Trans-Thoracic Echocardiography for the Diagnosis of Complex Congenital Heart Disease, PLoS One. Oct 2016; 11 (10).

12. Ou J, Srichai M, Hecht E, Kim D and Jacobs J.: Anatomy of the Heart at Multidetector CT: What the Radiologist Needs to Know, Radio Graphics, Nov/Dec 2007; volume 27;1569:1582.

13. Goo H., Park I., Ko J., et al. : State-of-theArt CT Imaging Techniques for Congenital Heart Disease. Korean J Radiol 2010; 11:418.

14. Bayraktutan U, Kantarci M, Ogul H, Kizrak Y, Pirimoglu B, Genc B, Yuceler Z and Ceviz N.: The utility of multidetector computed tomography for evaluation of congenital heart disease, May 2013, volume 72; 188:196.

15. Shehataa S, Zaitona F., Abo Wardaa M., Shahbahb D. and Ebrahim B.: Value of MDCT as a non- invasive modality in evaluation of pediatric congenital cardiovascular anomalies, The Egyptian Journal of Radiology and Nuclear Medicine, Volume 48, June 2017, Pages 467-478.

16. Seif El Dien H. , Ibrahim L. and Hashem R., Evaluation of Aortic Arch Anomalies by Echocardiography and CT Angiography, Could CT be the Primary Method of Diagnosis?, British Journal of Medicine \& Medical Research, March 2014, (16): 31793195

17. Kamala $\mathrm{O}$ A., Ahmed M M., Role of multidetector computed tomography 


\section{Dina Hamdy Ahmed, et al.,}

(MDCT) angiography in preoperative assessment of coarctation of the aorta in pediatric patients and young adults, The Egyptian Journal of Radiology and Nuclear Medicine, December 2011, Volume 42, Pages 297-303.
18. Osama A, Role of multi-slice CT angiography in the evaluation of pulmonary venous anomalies, The Egyptian Journal of Radiology and Nuclear Medicine, Volume 44, June 2013, Pages 193-201

$$
\begin{aligned}
& \text { تقييم الأثعة المقطعية متعددة المقاطع ذات الطاقة المنخفضة و المقترنة برسم القلب الكهربي لحالات } \\
& \text { عيوب القلب الخلقية لاى الاطفال } \\
& \text { د/ دينا حمدي أحمد و أ.د/ عبير مغاوري عبد الحميد و أ.د/ محمد أمين ناصف و د/ سوزان عادل علي عبد الرحيم } \\
& \text { ود/ عماد حامد عبدالدايم }
\end{aligned}
$$

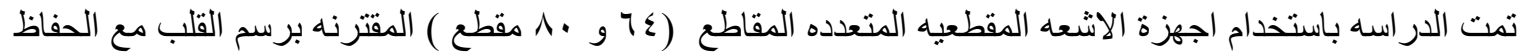

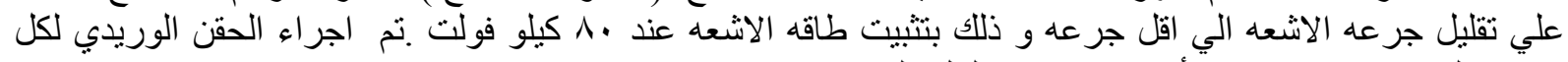

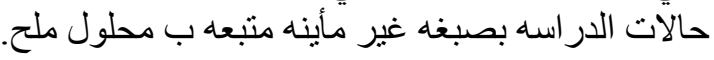

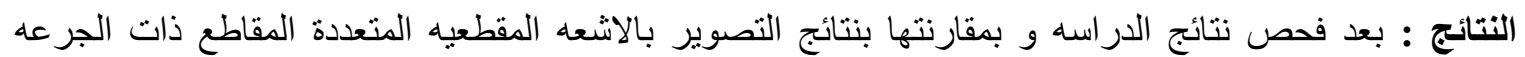

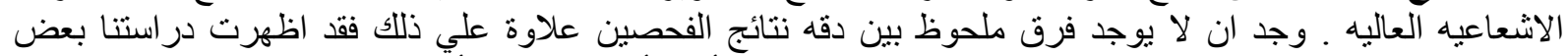

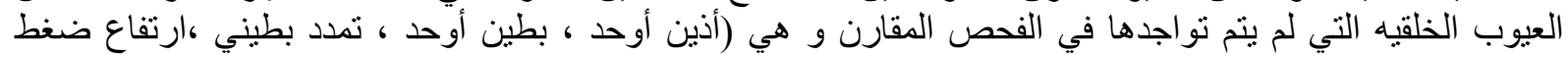
الثريان الرئوي).

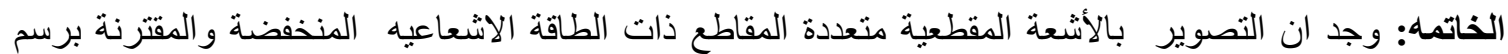

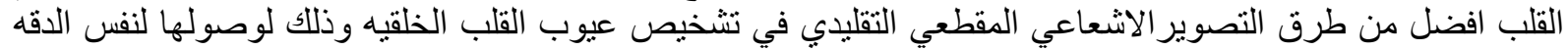

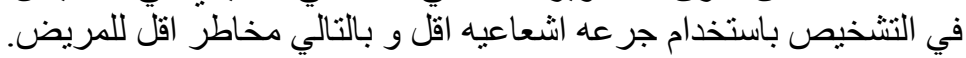

